\title{
Research on Short-term Ionospheric Prediction Combining with EOF and ARIMA Model Over Guangxi Area
}

\author{
Chen $\mathrm{Li}^{1}$, Hua Peng ${ }^{1}$, Liangke Huang ${ }^{1,2,3,}$, Lilong Liu ${ }^{1,2}$, Shaofeng Xie ${ }^{1,2}$ \\ 1. College of Geomatics and Geoinformation, Guilin University of Technology, Guilin 541004, China \\ 2. Guangxi Key Laboratory of Spatial Information and Geomatics, Guilin 541004, China \\ 3. GNSS Research Center, Wuhan Universtiy, Wuhan 430079, China
}

KEY WORDS: Ionosphere prediction； Empirical orthogonal function； ARIMA

\begin{abstract}
:
According to the empirical orthogonal function (EOF), the non-stationary time series data are decomposed into time function and space function, so this mathematical method can simplify the non-stationary time series and eliminate redundant information, thus it performs well in non-stationary time series analysis. The ionospheric Vertical Total Electron Content (VTEC) is a non-stationary time series, which has non-stationary and seasonal variation and the activity of VTEC is more active in low latitudes. Guangxi is located in the middle and low latitudes of the Northern Hemisphere with abundant sunshine in summer and autumn. The energy released by solar radiation makes the ionospheric activity in this region more complex than that in the high latitudes. However, no expert or scholar has used EOF analysis method to conduct a comprehensive study of the low latitudes. The International GNSS Service (IGS) provided by high precision Global Ionospheric Maps (GIM) center in Guangxi are used in the modeling data, the GIM data of the first 10 days of different seasons are decomposed by EOF, and then the time function is predicted by ARIMA model. VTEC values for the next five days are obtained through reconstruction, and relative accuracy and standard deviation are used as accuracy evaluation criteria. The results of EOF-ARIMA model are compared with those of ARIMA model, and the prediction accuracy of EOF-ARIMA model at the equatorial anomaly is analyzed in order to explore the reliability of the model in the more complex region of ionospheric activity. The results show that the average relative precision of EOF-ARIMA model is 84.0 , the average standard deviation is 7.45TECu, the average relative precision of ARIMA model is 81.5 , the average standard deviation is $8.29 \mathrm{TECu}$, and the precision of EOF-ARIMA model is higher than that of ARIMA model.; There is no significant seasonal difference in the prediction accuracy of EOF-ARIMA model, and the prediction accuracy of ARIMA model in autumn is lower than that of other seasons, which indicates that the prediction results of EOF-ARIMA model are more reliable; The prediction accuracy of the EOF-ARIMA model at the equatorial anomaly is not affected, and it is consistent with the accuracy of the high latitude area in Guangxi. It is shown that the EOF-ARIMA model has high accuracy and stability in the short-term ionospheric prediction in Guangxi at low latitudes of China, and provides a new and reliable method for ionospheric prediction at low latitudes.
\end{abstract}

\section{INTRODUCTION}

Total ionospheric electron content is an important parameter to characterize ionospheric delay. Improving the prediction accuracy of VTEC can improve the positioning accuracy of Global Navigation Satellite System (GNSS).In addition, it is also of great significance in the fields of pre-earthquake ionospheric disturbance, earth magnetic field research, and the influence of solar activity on the ionosphere [1-3].The commonly used VTEC prediction models mainly include grey model [4], neural network model [5], Holt-Winters model [6,7], ARMA model [8] and ARIMA model [9].Among them, the neural network model can be infinitely close to the complex non-linear relationship, and it can be well used in the prediction of VTEC. However, the network optimization is complex, the parameter selection is difficult, and the error of some predicted values is large, which limits its practical application to some extent. TEC prediction based on time series analysis has achieved great achievement $[8,9]$. However, using a single time series model directly to predict TEC will reduce its prediction accuracy. In order to improve the prediction accuracy of TEC, the literature [10] combined wavelet decomposition with ARIMA model to predict TEC, and the results showed that WARIMA model was feasible to predict ionospheric TEC, and the prediction accuracy was better than ARIMA model. It can be seen that pretreatment of TEC can improve the prediction accuracy of TEC.

EOF is a kind of mathematical analysis method, and the matrix can be decomposed into time function and space function. According to the variance contribution rate to simplify eliminate redundant information, EOF has been widely used in space and time characteristics and data analysis of non-stationary characteristics, such as: precipitation analysis [11], the average temperature anomaly value forecast [12], subsidence data analysis [13], and so on. However, few literatures have introduced EOF function into ARIMA model for short-term prediction of ionospheric VTEC in Guangxi region. The ionosphere in this area not only has equatorial anomalies [14], but also is a frequent area of typhoons, volcanoes and earthquakes $[15,16]$.

* Corresponding author: Liangke Huang 
However, the ionospheric VTEC will become abnormal before the occurrence of typhoons, volcanoes and earthquakes. High-precision VTEC prediction values can provide important data sources for the seismic prediction analysis [17] of Guangxi region and the analysis of the impact of typhoons on the ionosphere [18].Therefore, it is of great significance to explore the use of EOF- ARIMA model to predict ionospheric VTEC values in Guangxi region $\left(20^{\circ} \sim 27.5^{\circ} \mathrm{N}, 100^{\circ} \sim 115^{\circ} \mathrm{E}\right)$.

\section{INTRODUCTION TO MODEL PRINCIPLE}

\subsection{Introduction to EOF decomposition and refactoring fundamentals}

It has been shown in literature [9-11] that linear change of the original data can simplify the original data information and eliminate its redundant information. However, EOF decomposition is a common method, which can decompose the original matrix into two parts: space function matrix $\mathrm{V}$ and time function matrix Y. Among them, the spatial function partly depends on the main variation characteristics of the variable field and does not change with time. The decomposed time function is composed of the linear combination of the variables of the space points and is the main component. The specific process is as follows:

(1) Let the VTEC value with space-time characteristics be the matrix $\mathrm{Xij}$, the spatial function vik and the time function ykj, $\mathrm{k}=1,2 \ldots, \mathrm{m}$ has the following relationship:

$$
x_{i j}=\sum_{k=1}^{m} v_{i k} y_{k j}=v_{i 1} y_{1 j}+v_{i 2} y_{2 j}+\cdots+
$$

Its matrix representation is

$$
X=\left[\begin{array}{ccc}
v_{11} & \cdots & v_{1 m} \\
\vdots & \ddots & \vdots \\
v_{m 1} & \cdots & v_{m m}
\end{array}\right]\left[\begin{array}{ccc}
y_{11} & \cdots & \\
\vdots & \ddots & \\
y_{n 1} & \cdots &
\end{array}\right]
$$

(2) The variance contribution rate is calculated and the principal components with a contribution rate over $95 \%$ are selected for reconstruction, which can effectively eliminate the redundant information in the original sequence and ensure that the reconstructed spatio-temporal sequence can be obtained with relatively high accuracy. The variance contribution rate can be obtained by formula (3).

$$
R_{\mathrm{k}}=\lambda_{\mathrm{k}} / \sum_{i=1}^{n} \lambda_{\mathrm{i}}
$$

(3) The principal components that meet the requirements constitute matrix $\mathrm{PC}$ and are reconstructed through formula (4).

$$
X=V \cdot \mathrm{PC}
$$

\subsection{EOF - ARIMA modeling process}

Suppose a stationary time series is $\mathrm{xt}, \mathrm{t}=1,2,3 \ldots, \mathrm{N}$, ARMA has the following structure:

$$
\begin{gathered}
x_{\mathrm{q}}=\emptyset_{1} x_{\mathrm{i}-1}+\emptyset_{2} x_{\mathrm{i}-2}+\mathrm{K}+\emptyset_{\mathrm{p}} x_{\mathrm{i}-\mathrm{q}}+\varepsilon_{\mathrm{q}}+ \\
\theta_{1} \varepsilon_{\mathrm{t}-1}-\theta_{2} \varepsilon_{\mathrm{i}-2}-\mathrm{K}-\theta_{p} \varepsilon_{\mathrm{i}-q}+\varepsilon_{\mathrm{t}}
\end{gathered}
$$

(5)

Where $\mathrm{p}$ and $\mathrm{q}$ are seasonal or non-seasonal orders. ARIMA model is obtained by differential optimization of ARMA model and is suitable for forecasting non-stationary time series [19].

EOF - ARIMA modeling steps are as follows:

Step 1 (data preprocessing) in this study, the experimental data were used as the 2-hour resolution VTEC value of 16 grid points covering Guangxi region. Therefore, EOF decomposition was carried out on the data with the original matrix of $\mathrm{X} 308 \times 120$ of 16 grid points for 10 days in a season, and the time function $\mathrm{Y} 308 \times 120$ and spatial function $\mathrm{V} 121 \times 120$ were decomposed.

Step 2 (determination of order) carry out seasonal analysis on the principal components to determine the selection of seasonal prediction or non-seasonal prediction in ARIMA model. As the selected experimental samples of total ionospheric electron content have periodic changes with a period of 1 day, the seasonal prediction will be more accurate. The periodicity of the principal components is analyzed by sequence diagram to determine whether seasonal difference $\mathrm{D}$ or non-seasonal difference $\mathrm{D}$ is performed. The order of non-seasonal $p$ and $q$ and the order of seasonal $\mathrm{p}$ and $\mathrm{q}$ were determined by $\mathrm{ACF}$ and PACF.

Step 3 (prediction) determine the prediction days according to the experimental needs, and use the corresponding order to make seasonal prediction for the principal components.

Step 4 (reconstruction) construct the predicted principal components into matrix $\mathrm{PC}$, and reconstruct the predicted value of 5 days according to formula 6 .

\section{EXPERIMENTAL ANALYSIS}

\subsection{The data processing}

It has been shown in literature [9] that the factors affecting the prediction accuracy include sample size, days of prediction and the anomaly of ionosphere itself. Under the condition of the same sample size, the results show that the relative accuracy of the first 10 days is the highest, and the accuracy of the prediction after 30 days is significantly decreased. Under the condition that the forecast days are the same, when the sample number increases to more than 30 days, the prediction accuracy is not significantly improved. Therefore, in this paper, combined with the influence factors of prediction and the actual situation of Guangxi region, the global VTEC grid data of 2015 provided by IGS center were extracted to obtain the VTEC data of $2 \mathrm{~h}$ resolution of 16 grid points covering Guangxi region $\left(20^{\circ} \sim 27.5^{\circ} \mathrm{N}, 100^{\circ} \sim 115^{\circ} \mathrm{E}\right.$ ) (as shown in figure 1).Considering the periodic change of sunspot 11a [20], the VTEC values of 40 days (annual product days are 82 91 in spring, 184 193 in summer, 266 275 in autumn and 318 327 in winter respectively) of 10 days in each of the four seasons were selected as the modeling data, and the EOFARIMA model proposed in this paper was used to 
forecast the VTEC data of the next 5 days, and compared with the ARIMA model. Based on the VTEC value provided by IGS center as the reference value, standard deviation (STDd) and average relative accuracy (Ppd) were used to evaluate the prediction results of the model, and the formula was as follows:

$$
\begin{aligned}
S \mathrm{TD}_{\mathrm{d}} & =\sqrt{\frac{1}{12} \sum_{\mathrm{t}=1}^{12}\left(I_{\mathrm{pre}[\mathrm{t})}-\right.} \\
\mathrm{P}_{\mathrm{pd}} & =\frac{1}{12} \sum_{t=1}^{12}\left(1-\frac{I_{\mathrm{p}}}{12}\right.
\end{aligned}
$$

Where, Ipre $[\mathrm{t}]$ is the prediction result; Iigs $[\mathrm{t}]$ is the original sequence of VTEC provided by IGS center; $n$ represents the NTH day of the forecast days; STDd represents the daily standard deviation of the difference between the prediction results and the VTEC observations provided by IGS center. Ppd represents the average daily relative accuracy of the forecast results.

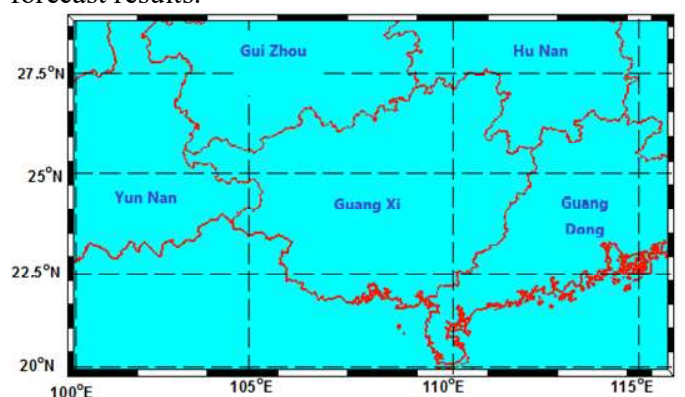

Figure 1 grid mesh distribution in Guangxi

\section{ANALYSIS OF EXPERIMENTAL RESULTS}

\subsection{Prediction accuracy analysis of EOF - ARIMA model}

As mentioned above, EOF - ARIMA model and ARIMA model were used to forecast and analyze the VTEC values of 16 grid points in 4 seasons covering Guangxi region. Due to the space limitation, this paper only selected 4 typical grid points in Guangxi region to analyze the prediction accuracy, and the results are shown in figure 2 to 5 . The $\mathrm{x}$-coordinate in figure 2 to 5 is the annual product day (d), which contains 12 VTEC values per day since IGS provides a time resolution of 2 hours. Ordinate is VTEC value, unit is $\mathrm{TECu}$; The blue square line segment represents the five-day VTEC measured value provided by IGS station, which is taken as the reference value in this paper. The red dotted line segment represents the VTEC value predicted by EOF-ARIMA model for 5 days.

From figure 2 to figure 5 , it can be seen that in the four seasons of the selected four grid points, EOF-ARIMA model and the measured values provided by IGS center have a good consistency, indicating that EOF-ARIMA model has superior prediction performance. However, when the VTEC value in the sample data fluctuates greatly, such as $22.5^{\circ} \mathrm{N}, 105^{\circ} \mathrm{E}$ spring, $25^{\circ} \mathrm{N}, 110^{\circ} \mathrm{E}$ spring, and $27.5^{\circ} \mathrm{N}$ and $105^{\circ} \mathrm{E}$ spring, the prediction accuracy decreases.
The main reason may be the defect of ARIMA model itself, which has poor fitting effect in the face of complex and irregular time series. Nevertheless, the EOF-ARIMA model combined with EOF still shows superiority in forecasting performance, especially in low latitude grid points in Guangxi.



Figure $2\left(20^{\circ} \mathrm{N}, 100^{\circ} \mathrm{E}\right) \mathrm{VTEC}$ value of grid node

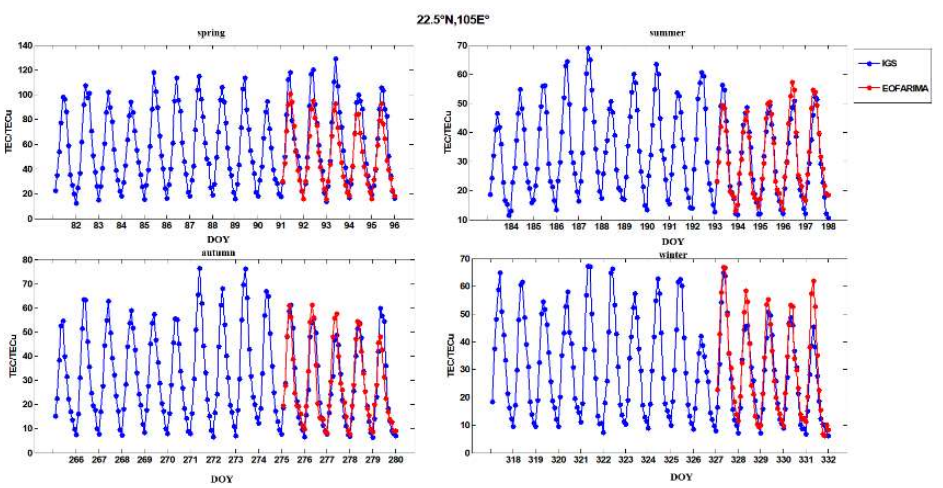

Figure $3\left(22.5^{\circ} \mathrm{N}, 105^{\circ} \mathrm{E}\right) \mathrm{VTEC}$ value of grid node
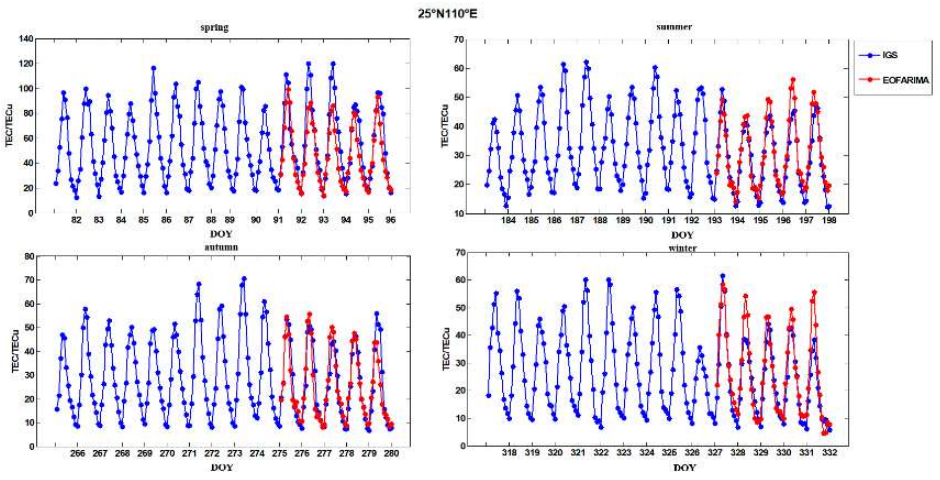

Figure $4\left(25^{\circ} \mathrm{N}, 110^{\circ} \mathrm{E}\right) \mathrm{VTEC}$ value of grid node 

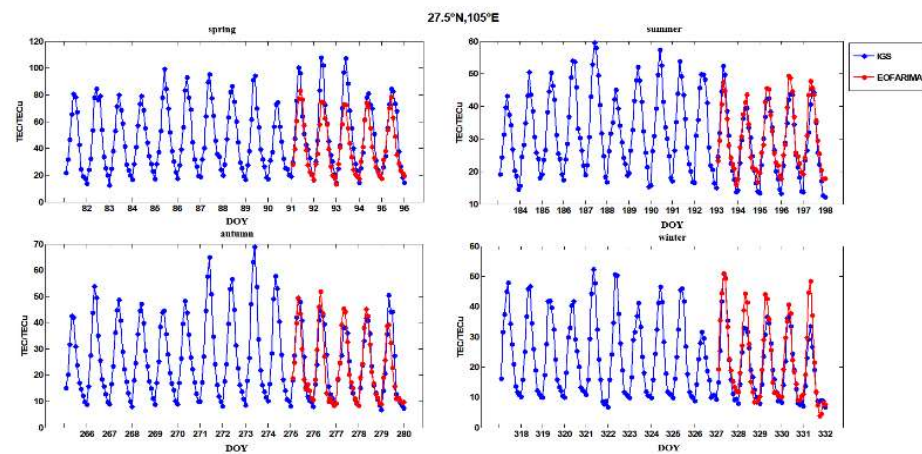

Figure $5 \quad\left(27.5^{\circ} \mathrm{N}, 105^{\circ} \mathrm{E}\right) \mathrm{VTEC}$ value of grid node

In order to deeply analyze the prediction effect of EOF-ARIMA model in Guangxi region, the prediction accuracy of two models representing 6 character nodes in Guangxi region was also statistically analyzed. The results are shown in table 1.

As can be seen from table 1 , in the same season, the relative accuracy and standard deviation of EOF-ARIMA model remain in the same prediction accuracy and are more stable than ARIMA model, which further indicates that EOF-ARIMA model has better stability in the short-term VTEC prediction in Guangxi region. In conclusion, the average relative accuracy and mean standard deviation of EOF-ARIMA model for VTEC prediction in Guangxi region are $84.0 \%$ and 7.45 TECu respectively, which can maintain good stability in different seasons, indicating that the introduction of EOF decomposition into ARIMA model can improve the short-term prediction accuracy of ARIMA model for VTEC.

Table 1 VTEC prediction accuracy statistics of the two models in 4 representative character nodes in Guangxi region

\begin{tabular}{|c|c|c|c|c|c|c|c|c|c|c|c|c|c|c|}
\hline \multirow{2}{*}{$\begin{array}{c}\text { seaso } \\
\mathrm{n}\end{array}$} & \multirow{2}{*}{ grid } & \multirow{2}{*}{ model } & \multicolumn{10}{|c|}{ Days (relative accuracy standard deviation) } & \multirow{2}{*}{\multicolumn{2}{|c|}{ mean }} \\
\hline & & & \multicolumn{2}{|c|}{1} & \multicolumn{2}{|c|}{2} & \multicolumn{2}{|c|}{3} & \multicolumn{2}{|c|}{4} & \multicolumn{2}{|c|}{5} & & \\
\hline \multirow{10}{*}{$\begin{array}{c}\text { sprin } \\
\mathrm{g}\end{array}$} & \multirow{2}{*}{$\begin{array}{l}20^{\circ} \mathrm{N} \\
100^{\circ} \mathrm{E}\end{array}$} & $\begin{array}{c}\text { W-ARI } \\
\text { MA }\end{array}$ & $\begin{array}{c}90.6 \\
\%\end{array}$ & 6.07 & $\begin{array}{c}87.1 \\
\%\end{array}$ & 6.44 & $\begin{array}{c}85.3 \\
\%\end{array}$ & 7.56 & $\begin{array}{c}90.5 \\
\%\end{array}$ & 7.09 & $\begin{array}{c}90.6 \\
\%\end{array}$ & 8.10 & $\begin{array}{c}88.8 \\
\%\end{array}$ & 7.05 \\
\hline & & $\begin{array}{l}\text { EOF-A } \\
\text { RIMA }\end{array}$ & $\begin{array}{c}91.9 \\
\%\end{array}$ & 8.16 & $\begin{array}{c}84.7 \\
\%\end{array}$ & 9.37 & $\begin{array}{c}80.5 \\
\%\end{array}$ & $\begin{array}{c}11.6 \\
1\end{array}$ & $\begin{array}{c}82.5 \\
\%\end{array}$ & $\begin{array}{c}11.6 \\
0\end{array}$ & $\begin{array}{c}85.7 \\
\%\end{array}$ & $\begin{array}{c}11.5 \\
1\end{array}$ & $\begin{array}{c}85.1 \\
\%\end{array}$ & $\begin{array}{c}10.4 \\
5\end{array}$ \\
\hline & \multirow{3}{*}{$\begin{array}{c}22.5^{\circ} \mathrm{N} \\
105^{\circ} \mathrm{E}\end{array}$} & $\begin{array}{c}\text { W-ARI } \\
\text { MA }\end{array}$ & $\begin{array}{c}88.5 \\
\%\end{array}$ & 7.45 & $\begin{array}{c}82.9 \\
\%\end{array}$ & 9.16 & $\begin{array}{c}79.5 \\
\%\end{array}$ & $\begin{array}{c}11.0 \\
8\end{array}$ & $\begin{array}{c}89.8 \\
\%\end{array}$ & 8.34 & $\begin{array}{c}84.1 \\
\%\end{array}$ & $\begin{array}{c}13.1 \\
9\end{array}$ & $\begin{array}{c}85.0 \\
\%\end{array}$ & 9.84 \\
\hline & & EOF-A & 87.7 & 10.2 & 85.3 & 10.5 & 75.6 & 12.9 & 82.5 & 11.3 & 84.2 & 12.4 & 83.0 & 11.5 \\
\hline & & RIMA & $\%$ & 5 & $\%$ & 5 & $\%$ & 8 & $\%$ & 1 & $\%$ & 1 & $\%$ & 0 \\
\hline & \multirow{3}{*}{$\begin{array}{l}25^{\circ} \mathrm{N} \\
110^{\circ} \mathrm{E}\end{array}$} & $\begin{array}{c}\text { W-ARI } \\
\text { MA }\end{array}$ & $\begin{array}{c}87.1 \\
\%\end{array}$ & 8.84 & $\begin{array}{c}80.3 \\
\%\end{array}$ & $\begin{array}{c}11.2 \\
4\end{array}$ & $\begin{array}{c}76.9 \\
\%\end{array}$ & $\begin{array}{c}11.6 \\
5\end{array}$ & $\begin{array}{c}90.8 \\
\%\end{array}$ & 6.88 & $\begin{array}{c}79.9 \\
\%\end{array}$ & $\begin{array}{c}14.5 \\
7\end{array}$ & $\begin{array}{c}83.0 \\
\%\end{array}$ & $\begin{array}{c}10.6 \\
4\end{array}$ \\
\hline & & EOF-A & 84.3 & 11.6 & 83.9 & 12.4 & 72.3 & 12.8 & 82.6 & 10.1 & 83.4 & 13.4 & 81.3 & 12.1 \\
\hline & & RIMA & $\%$ & 2 & $\%$ & 4 & $\%$ & 5 & $\%$ & 9 & $\%$ & 0 & $\%$ & 0 \\
\hline & \multirow{2}{*}{$\begin{array}{c}27.5^{\circ} \mathrm{N} \\
105^{\circ} \mathrm{E}\end{array}$} & $\begin{array}{c}\text { W-ARI } \\
\text { MA }\end{array}$ & $\begin{array}{c}86.0 \\
\%\end{array}$ & 7.58 & $\begin{array}{c}81.7 \\
\%\end{array}$ & $\begin{array}{c}10.2 \\
7\end{array}$ & $\begin{array}{c}78.5 \\
\%\end{array}$ & 10.9 & $\begin{array}{c}87.6 \\
\%\end{array}$ & 7.40 & $\begin{array}{c}80.5 \\
\%\end{array}$ & $\begin{array}{c}12.3 \\
0\end{array}$ & $\begin{array}{c}82.8 \\
\%\end{array}$ & 9.69 \\
\hline & & $\begin{array}{l}\text { EOF-A } \\
\text { RIMA }\end{array}$ & $\begin{array}{c}84.5 \\
\%\end{array}$ & 9.51 & $\begin{array}{c}81.3 \\
\%\end{array}$ & $\begin{array}{c}10.6 \\
5\end{array}$ & $\begin{array}{c}74.2 \\
\%\end{array}$ & $\begin{array}{c}12.8 \\
1\end{array}$ & $\begin{array}{c}85.7 \\
\%\end{array}$ & 9.82 & $\begin{array}{c}80.4 \\
\%\end{array}$ & $\begin{array}{c}11.9 \\
6\end{array}$ & $\begin{array}{c}81.2 \\
\%\end{array}$ & $\begin{array}{c}10.9 \\
5\end{array}$ \\
\hline \multirow{8}{*}{$\begin{array}{l}\text { sum } \\
\text { mer }\end{array}$} & \multirow{2}{*}{$\begin{array}{l}20^{\circ} \mathrm{N} \\
100^{\circ} \mathrm{E}\end{array}$} & $\begin{array}{c}\text { W-ARI } \\
\text { MA }\end{array}$ & $\begin{array}{c}80.2 \\
\%\end{array}$ & 4.73 & $\begin{array}{c}79.3 \\
\%\end{array}$ & 3.20 & $\begin{array}{c}79.7 \\
\%\end{array}$ & 2.32 & $\begin{array}{c}82.1 \\
\%\end{array}$ & 2.23 & $\begin{array}{c}81.4 \\
\%\end{array}$ & 2.24 & $\begin{array}{c}80.5 \\
\%\end{array}$ & 2.95 \\
\hline & & $\begin{array}{l}\text { EOF-A } \\
\text { RIMA }\end{array}$ & $\begin{array}{c}88.2 \\
\%\end{array}$ & 4.41 & $\begin{array}{c}84.6 \\
\%\end{array}$ & 4.00 & $\begin{array}{c}89.3 \\
\%\end{array}$ & 2.55 & $\begin{array}{c}85.7 \\
\%\end{array}$ & 1.81 & $\begin{array}{c}75.9 \\
\%\end{array}$ & 3.42 & $\begin{array}{c}84.8 \\
\%\end{array}$ & 3.24 \\
\hline & \multirow{2}{*}{$\begin{array}{c}22.5^{\circ} \mathrm{N} \\
105^{\circ} \mathrm{E}\end{array}$} & $\begin{array}{c}\text { W-ARI } \\
\text { MA }\end{array}$ & $\begin{array}{c}82.1 \\
\%\end{array}$ & 4.21 & $\begin{array}{c}77.1 \\
\%\end{array}$ & 3.26 & $\begin{array}{c}80.6 \\
\%\end{array}$ & 2.20 & $\begin{array}{c}80.2 \\
\%\end{array}$ & 2.26 & $\begin{array}{c}82.9 \\
\%\end{array}$ & 2.44 & $\begin{array}{c}80.6 \\
\%\end{array}$ & 2.87 \\
\hline & & $\begin{array}{l}\text { EOF-A } \\
\text { RIMA }\end{array}$ & $\begin{array}{c}89.6 \\
\%\end{array}$ & 3.35 & $\begin{array}{c}86.5 \\
\%\end{array}$ & 3.63 & $\begin{array}{c}89.5 \\
\%\end{array}$ & 2.46 & $\begin{array}{c}84.5 \\
\%\end{array}$ & 2.68 & $\begin{array}{c}77.8 \\
\%\end{array}$ & 3.60 & $\begin{array}{c}85.6 \\
\%\end{array}$ & 3.15 \\
\hline & \multirow{2}{*}{$\begin{array}{l}25^{\circ} \mathrm{N} \\
110^{\circ} \mathrm{E}\end{array}$} & $\begin{array}{c}\text { W-ARI } \\
\text { MA }\end{array}$ & $\begin{array}{l}77.8 \\
\%\end{array}$ & 4.73 & $\begin{array}{c}73.4 \\
\%\end{array}$ & 3.34 & $\begin{array}{c}78.0 \\
\%\end{array}$ & 2.37 & $\begin{array}{c}77.9 \\
\%\end{array}$ & 1.64 & $\begin{array}{c}79.5 \\
\%\end{array}$ & 1.80 & $\begin{array}{c}77.3 \\
\%\end{array}$ & 2.78 \\
\hline & & $\begin{array}{l}\text { EOF-A } \\
\text { RIMA }\end{array}$ & $\begin{array}{c}90.8 \\
\%\end{array}$ & 2.45 & $\begin{array}{c}87.2 \\
\%\end{array}$ & 3.31 & $\begin{array}{c}87.5 \\
\%\end{array}$ & 2.87 & $\begin{array}{c}82.4 \\
\%\end{array}$ & 3.46 & $\begin{array}{c}80.7 \\
\%\end{array}$ & 3.78 & $\begin{array}{c}85.7 \\
\%\end{array}$ & 3.17 \\
\hline & \multirow{2}{*}{$\begin{array}{c}27.5^{\circ} \mathrm{N} \\
105^{\circ} \mathrm{E}\end{array}$} & $\begin{array}{c}\text { W-ARI } \\
\text { MA }\end{array}$ & $\begin{array}{c}84.9 \\
\%\end{array}$ & 3.36 & $\begin{array}{c}74.4 \\
\%\end{array}$ & 2.98 & $\begin{array}{c}81.5 \\
\%\end{array}$ & 1.61 & $\begin{array}{c}80.8 \\
\%\end{array}$ & 1.37 & $\begin{array}{c}78.7 \\
\%\end{array}$ & 2.67 & $\begin{array}{c}80.0 \\
\%\end{array}$ & 2.40 \\
\hline & & $\begin{array}{l}\text { EOF-A } \\
\text { RIMA }\end{array}$ & $\begin{array}{c}88.8 \\
\% \\
\end{array}$ & 3.09 & $\begin{array}{c}85.3 \\
\% \\
\end{array}$ & 3.31 & $\begin{array}{c}86.6 \\
\%\end{array}$ & 2.45 & $\begin{array}{c}84.0 \\
\%\end{array}$ & 2.24 & $\begin{array}{c}82.1 \\
\%\end{array}$ & 3.26 & $\begin{array}{c}85.4 \\
\%\end{array}$ & 2.87 \\
\hline \multirow[t]{7}{*}{$\begin{array}{c}\text { autu } \\
\text { mn }\end{array}$} & \multirow{2}{*}{$\begin{array}{l}20^{\circ} \mathrm{N} \\
100^{\circ} \mathrm{E}\end{array}$} & $\begin{array}{c}\text { W-ARI } \\
\text { MA }\end{array}$ & $\begin{array}{c}89.5 \\
\%\end{array}$ & 1.58 & $\begin{array}{c}77.4 \\
\%\end{array}$ & 2.96 & $\begin{array}{c}67.9 \\
\%\end{array}$ & 5.00 & $\begin{array}{c}78.3 \\
\%\end{array}$ & 3.45 & $\begin{array}{c}68.8 \\
\%\end{array}$ & 3.19 & $\begin{array}{c}76.4 \\
\%\end{array}$ & 3.23 \\
\hline & & $\begin{array}{c}\text { EOF-A } \\
\text { RIMA }\end{array}$ & $\begin{array}{c}83.7 \\
\%\end{array}$ & 5.05 & $\begin{array}{c}81.7 \\
\%\end{array}$ & 7.11 & $\begin{array}{c}75.2 \\
\%\end{array}$ & 5.39 & $\begin{array}{c}85.6 \\
\%\end{array}$ & 3.51 & $\begin{array}{c}74.6 \\
\%\end{array}$ & 8.50 & $\begin{array}{c}80.1 \\
\%\end{array}$ & 5.91 \\
\hline & \multirow{2}{*}{$\begin{array}{c}22.5^{\circ} \mathrm{N} \\
105^{\circ} \mathrm{E}\end{array}$} & $\begin{array}{c}\text { W-ARI } \\
\text { MA }\end{array}$ & $\begin{array}{c}78.0 \\
\%\end{array}$ & 2.66 & $\begin{array}{c}79.3 \\
\%\end{array}$ & 3.37 & $\begin{array}{c}63.0 \\
\%\end{array}$ & 6.60 & $\begin{array}{c}76.0 \\
\%\end{array}$ & 4.43 & $\begin{array}{c}65.3 \\
\%\end{array}$ & 3.53 & $\begin{array}{c}72.3 \\
\%\end{array}$ & 4.12 \\
\hline & & $\begin{array}{l}\text { EOF-A } \\
\text { RIMA }\end{array}$ & $\begin{array}{c}82.2 \\
\%\end{array}$ & 5.34 & $\begin{array}{c}82.1 \\
\%\end{array}$ & 7.18 & $\begin{array}{c}77.1 \\
\%\end{array}$ & 5.44 & $\begin{array}{c}86.7 \\
\%\end{array}$ & 3.14 & $\begin{array}{c}75.2 \\
\%\end{array}$ & 9.16 & $\begin{array}{c}80.7 \\
\%\end{array}$ & 6.06 \\
\hline & \multirow{2}{*}{$\begin{array}{l}25^{\circ} \mathrm{N} \\
110^{\circ} \mathrm{E}\end{array}$} & $\begin{array}{c}\text { W-ARI } \\
\text { MA }\end{array}$ & $\begin{array}{c}73.5 \\
\%\end{array}$ & 3.76 & $\begin{array}{c}83.4 \\
\%\end{array}$ & 3.06 & $\begin{array}{c}63.0 \\
\%\end{array}$ & 6.94 & $\begin{array}{c}73.4 \\
\%\end{array}$ & 4.19 & $\begin{array}{c}65.3 \\
\%\end{array}$ & 3.58 & $\begin{array}{c}71.7 \\
\%\end{array}$ & 4.31 \\
\hline & & $\begin{array}{l}\text { EOF-A } \\
\text { RIMA }\end{array}$ & $\begin{array}{c}81.5 \\
\%\end{array}$ & 5.40 & $\begin{array}{c}82.5 \\
\%\end{array}$ & 6.93 & $\begin{array}{c}80.4 \\
\%\end{array}$ & 5.41 & $\begin{array}{c}87.0 \\
\%\end{array}$ & 2.76 & $\begin{array}{c}76.3 \\
\%\end{array}$ & 9.05 & $\begin{array}{c}81.6 \\
\%\end{array}$ & 5.91 \\
\hline & $27.5^{\circ} \mathrm{N}$ & W-ARI & 81.7 & 4.48 & 87.6 & 4.45 & 70.0 & 7.86 & 74.1 & 4.81 & 68.1 & 4.18 & 76.3 & 5.15 \\
\hline
\end{tabular}




\begin{tabular}{|c|c|c|c|c|c|c|c|c|c|c|c|c|c|c|}
\hline & \multirow[b]{2}{*}{$105^{\circ} \mathrm{E}$} & MA & $\%$ & \multicolumn{3}{|c|}{$\%$} & $\%$ & \multicolumn{3}{|c|}{$\%$} & $\%$ & \multicolumn{3}{|c|}{$\%$} \\
\hline & & $\begin{array}{c}\text { EOF-A } \\
\text { RIMA }\end{array}$ & $\begin{array}{c}82.1 \\
\% \\
\end{array}$ & 4.57 & $\begin{array}{c}81.2 \\
\% \\
\end{array}$ & 6.48 & $\begin{array}{c}87.5 \\
\% \\
\end{array}$ & 4.42 & $\begin{array}{c}89.0 \\
\% \\
\end{array}$ & 2.45 & $\begin{array}{c}76.7 \\
\% \\
\end{array}$ & 8.13 & $\begin{array}{c}83.3 \\
\% \\
\end{array}$ & 5.21 \\
\hline \multirow{8}{*}{$\begin{array}{l}\text { winte } \\
r\end{array}$} & \multirow{2}{*}{$\begin{array}{c}20^{\circ} \mathrm{N} \\
100^{\circ} \mathrm{E}\end{array}$} & $\begin{array}{c}\text { W-ARI } \\
\text { MA }\end{array}$ & $\begin{array}{c}92.5 \\
\%\end{array}$ & 2.66 & $\begin{array}{c}88.1 \\
\%\end{array}$ & 4.67 & $\begin{array}{c}87.7 \\
\%\end{array}$ & 3.33 & $\begin{array}{c}83.4 \\
\%\end{array}$ & 4.40 & $\begin{array}{c}69.1 \\
\%\end{array}$ & 7.23 & $\begin{array}{c}84.1 \\
\%\end{array}$ & 4.46 \\
\hline & & $\begin{array}{c}\text { EOF-A } \\
\text { RIMA }\end{array}$ & $\begin{array}{c}70.0 \\
\%\end{array}$ & 3.30 & $\begin{array}{c}83.5 \\
\%\end{array}$ & 5.31 & $\begin{array}{c}83.4 \\
\%\end{array}$ & 5.67 & $\begin{array}{c}77.7 \\
\%\end{array}$ & 3.08 & $\begin{array}{c}71.0 \\
\%\end{array}$ & 5.94 & $\begin{array}{c}77.1 \\
\%\end{array}$ & 4.66 \\
\hline & \multirow{2}{*}{$\begin{array}{l}22.5^{\circ} \mathrm{N} \\
105^{\circ} \mathrm{E}\end{array}$} & $\begin{array}{c}\text { W-ARI } \\
\text { MA }\end{array}$ & $\begin{array}{c}86.5 \\
\%\end{array}$ & 7.90 & $\begin{array}{c}87.4 \\
\%\end{array}$ & 3.90 & $\begin{array}{c}80.5 \\
\%\end{array}$ & 1.30 & $\begin{array}{c}85.3 \\
\%\end{array}$ & 4.63 & $\begin{array}{c}75.0 \\
\%\end{array}$ & 6.01 & $\begin{array}{c}82.9 \\
\%\end{array}$ & 4.75 \\
\hline & & $\begin{array}{c}\text { EOF-A } \\
\text { RIMA }\end{array}$ & $\begin{array}{c}74.3 \\
\%\end{array}$ & 3.07 & $\begin{array}{c}80.9 \\
\%\end{array}$ & 5.74 & $\begin{array}{c}84.3 \\
\%\end{array}$ & 5.08 & $\begin{array}{c}75.7 \\
\%\end{array}$ & 3.02 & $\begin{array}{c}67.1 \\
\%\end{array}$ & 7.07 & $\begin{array}{c}76.5 \\
\%\end{array}$ & 4.80 \\
\hline & \multirow{2}{*}{$\begin{array}{l}25^{\circ} \mathrm{N} \\
110^{\circ} \mathrm{E}\end{array}$} & $\begin{array}{c}\text { W-ARI } \\
\text { MA }\end{array}$ & $\begin{array}{c}84.8 \\
\%\end{array}$ & 8.51 & $\begin{array}{c}83.9 \\
\%\end{array}$ & 4.28 & $\begin{array}{c}82.8 \\
\%\end{array}$ & 1.46 & $\begin{array}{c}83.5 \\
\%\end{array}$ & 4.78 & $\begin{array}{c}72.4 \\
\%\end{array}$ & 5.29 & $\begin{array}{c}81.5 \\
\%\end{array}$ & 4.86 \\
\hline & & $\begin{array}{c}\text { EOF-A } \\
\text { RIMA }\end{array}$ & $\begin{array}{c}75.3 \\
\%\end{array}$ & 3.60 & $\begin{array}{c}76.4 \\
\%\end{array}$ & 6.42 & $\begin{array}{c}83.3 \\
\%\end{array}$ & 4.14 & $\begin{array}{c}74.1 \\
\%\end{array}$ & 3.03 & $\begin{array}{c}66.9 \\
\%\end{array}$ & 7.09 & $\begin{array}{c}75.2 \\
\%\end{array}$ & 4.86 \\
\hline & \multirow{2}{*}{$\begin{array}{l}27.5^{\circ} \mathrm{N} \\
105^{\circ} \mathrm{E}\end{array}$} & $\begin{array}{c}\text { W-ARI } \\
\text { MA }\end{array}$ & $\begin{array}{c}87.2 \\
\%\end{array}$ & 6.60 & $\begin{array}{c}86.0 \\
\%\end{array}$ & 3.59 & $\begin{array}{c}87.1 \\
\%\end{array}$ & 0.89 & $\begin{array}{c}89.8 \\
\%\end{array}$ & 3.40 & $\begin{array}{c}77.0 \\
\%\end{array}$ & 4.25 & $\begin{array}{c}85.4 \\
\%\end{array}$ & 3.75 \\
\hline & & $\begin{array}{l}\text { EOF-A } \\
\text { RIMA }\end{array}$ & $\begin{array}{c}78.6 \\
\%\end{array}$ & 2.96 & $\begin{array}{c}78.6 \\
\%\end{array}$ & 5.08 & $\begin{array}{c}81.0 \\
\%\end{array}$ & 4.76 & $\begin{array}{c}80.6 \\
\%\end{array}$ & 2.72 & $\begin{array}{c}71.2 \\
\%\end{array}$ & 6.63 & $\begin{array}{c}78.0 \\
\%\end{array}$ & 4.43 \\
\hline
\end{tabular}

\subsection{Analysis of prediction accuracy in different latitudes}

The ionospheric equatorial anomaly is caused by the movement of ionospheric electrons near the equator along the magnetic field line to both ends of high latitude, forming the phenomenon of hump in the northern hemisphere. The northern hump mainly occurs in the geomagnetic latitude $10^{\circ} \sim 12.5^{\circ} \mathrm{N}$ (the geographic latitude is about $20^{\circ} \sim 22.5^{\circ} \mathrm{N}$ ) [21]. Territory is vast in China, stretching from north to south across the low, middle and high latitudes. The maximum daily peak value of all seasons occurs in the low latitudes, and the maximum daily peak value is much higher than the average daily average value of China. Due to equatorial anomalies in the ionosphere at low latitudes, it is necessary to further analyze the applicability of EOF-ARIMA model at different latitudes in China, especially at low latitudes in China. In this paper, 8 grid nodes $\left(5^{\circ} \mathrm{N}, 110^{\circ} \mathrm{E}, 15^{\circ} \mathrm{N}, 110^{\circ} \mathrm{E}\right.$, $17.5^{\circ} \mathrm{N}, 110^{\circ} \mathrm{E}, \quad 20^{\circ} \mathrm{N}, 110^{\circ} \mathrm{E}, \quad 22.5^{\circ} \mathrm{N}, 110^{\circ} \mathrm{E}$, $30^{\circ} \mathrm{N}, 110^{\circ} \mathrm{E}, \quad 40^{\circ} \mathrm{N}, 110^{\circ} \mathrm{E}, \quad 50^{\circ} \mathrm{N}, 110^{\circ} \mathrm{E}, 110^{\circ} \mathrm{E}$, $50^{\circ} \mathrm{N}, 110^{\circ} \mathrm{E}$ ) at different latitudes in China were selected to analyze their prediction accuracy at different latitudes. The 10-day VTEC values of 8 grid points in summer were selected as the modeling data (as shown in figure 6), and EOF-ARIMA model was used to predict the data of 184-193 (summer) annual plot days for the next 5 days. The prediction results were shown in table 2 .

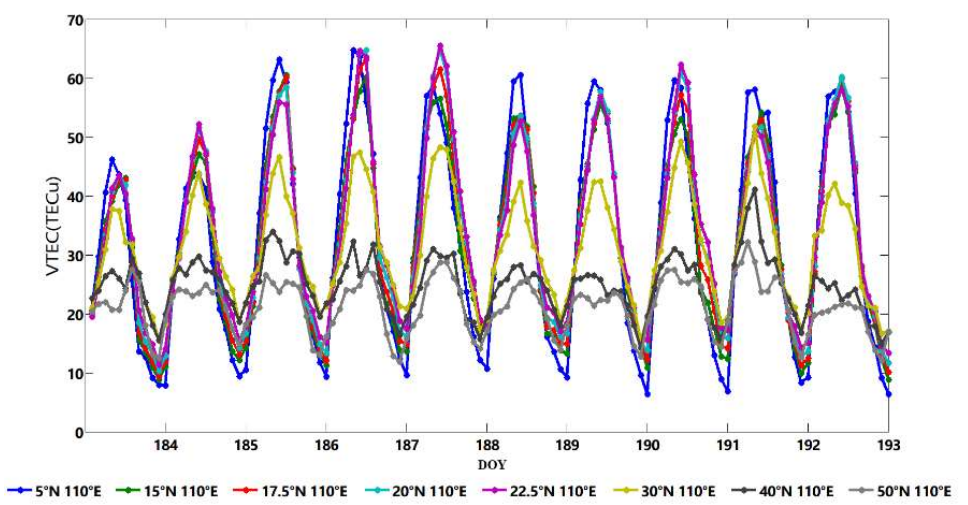

fig. 6 measured VTEC of IGS stations at middle, low and high latitudes in China

Table 2 prediction accuracy statistics of eof-arima model at different latitudes in China

\begin{tabular}{|c|c|c|c|c|c|c|c|c|c|c|c|c|}
\hline \multirow[b]{2}{*}{ grid } & \multicolumn{2}{|c|}{1} & \multicolumn{2}{|c|}{2} & \multicolumn{2}{|c|}{3} & \multicolumn{2}{|c|}{4} & \multicolumn{2}{|c|}{5} & \multicolumn{2}{|c|}{ mean } \\
\hline & $\begin{array}{c}\text { STD } \\
(\mathrm{TECu})\end{array}$ & $\begin{array}{c}\mathrm{P} \\
(\%)\end{array}$ & $\begin{array}{c}\text { STD } \\
(\mathrm{TECu})\end{array}$ & $\begin{array}{c}\mathrm{P} \\
(\%)\end{array}$ & $\begin{array}{c}\text { STD } \\
(\mathrm{TECu})\end{array}$ & $\begin{array}{c}\mathrm{P} \\
(\%)\end{array}$ & $\begin{array}{c}\text { STD } \\
(\mathrm{TECu})\end{array}$ & $\begin{array}{c}\mathrm{P} \\
(\%)\end{array}$ & $\begin{array}{c}\text { STD } \\
(\mathrm{TECu})\end{array}$ & $\begin{array}{c}\mathrm{P} \\
(\%)\end{array}$ & $\begin{array}{c}\text { STD } \\
(\mathrm{TECu})\end{array}$ & $\begin{array}{c}\mathrm{P} \\
(\%)\end{array}$ \\
\hline $5^{\circ} \mathrm{N}, 110^{\circ} \mathrm{E}$ & 3.84 & 83.3 & 3.84 & 86.8 & 2.70 & 90.7 & 3.33 & 86.8 & 4.60 & 82.9 & 3.66 & 86.1 \\
\hline $15^{\circ} \mathrm{N}, 110^{\circ} \mathrm{E}$ & 3.38 & 87.1 & 2.36 & 93.6 & 4.24 & 88.7 & 2.79 & 87.5 & 3.99 & 79.3 & 3.35 & 87.2 \\
\hline $17.5^{\circ} \mathrm{N}, 110^{\circ} \mathrm{E}$ & 3.58 & 88.9 & 2.69 & 93.8 & 4.05 & 89.4 & 2.88 & 85.1 & 2.62 & 79.3 & 3.16 & 87.3 \\
\hline
\end{tabular}




\begin{tabular}{ccccccccccccc}
$20^{\circ} \mathrm{N}, 110^{\circ} \mathrm{E}$ & 3.73 & 90.8 & 3.40 & 89.7 & 3.62 & 90.4 & 3.03 & 81.7 & 1.77 & 77.2 & 3.11 & 86.0 \\
$22.5^{\circ} \mathrm{N}, 110^{\circ} \mathrm{E}$ & 3.68 & 92.1 & 3.98 & 83.5 & 3.02 & 91.5 & 2.90 & 79.2 & 1.80 & 76.3 & 3.08 & 84.5 \\
$30^{\circ} \mathrm{N}, 110^{\circ} \mathrm{E}$ & 2.70 & 89.4 & 3.84 & 70.5 & 1.47 & 83.0 & 1.33 & 81.7 & 2.08 & 80.0 & 2.28 & 80.9 \\
$40^{\circ} \mathrm{N}, 110^{\circ} \mathrm{E}$ & 3.13 & 86.8 & 1.90 & 67.2 & 2.34 & 77.4 & 1.68 & 90.2 & 2.25 & 81.2 & 2.26 & 80.6 \\
$50^{\circ} \mathrm{N}, 110^{\circ} \mathrm{E}$ & 3.21 & 80.5 & 2.29 & 69.4 & 1.97 & 91.9 & 1.46 & 90.4 & 1.66 & 82.6 & 2.12 & 82.9 \\
\hline
\end{tabular}

As can be seen from figure 6, VTEC value in the region of $15^{\circ} \sim 22.5^{\circ} \mathrm{N}$ is higher than that in other latitudes, and has no correlation with latitudes. In the region of $22.5^{\circ} \sim 50^{\circ} \mathrm{N}$, VTEC value decreases with the increase of latitudes due to less solar radiation, which indicates that ionospheric equatorial anomalies exist in China's low latitudes. As can be seen from table 3, the relative accuracy of EOF-ARIMA model in VTEC prediction of different latitudes in China is above $80 \%$, and the standard deviation does not fluctuate greatly. The relative accuracy in the region of $5^{\circ} \sim 22.5^{\circ} \mathrm{N}$ is above $84 \%$, indicating that the short-term prediction accuracy of EOF-ARIMA model is not affected by the ionospheric equatorial anomaly. On the whole, the average relative accuracy of EOF-ARIMA model in different latitudes in China is higher than $80 \%$, indicating that EOF can eliminate redundant information by preprocessing data. By introducing EOF decomposition, the short-term prediction ability of ARIMA model VTEC can be improved, so that EOF-ARIMA model can achieve higher short-term prediction accuracy of VTEC in China. Therefore, EOF-ARIMA model has good applicability and stability in short-term prediction of VTEC in China.

\section{CONCLUSION}

Based on the function of EOF to simplify non-stationary time series and eliminate redundant information, this paper introduces EOF into ARIMA model to obtain the combined model EOF-ARIMA. Based on the VTEC data of 308 grid points with 2-hour resolution covering Guangxi in 2015 provided by IGS center, the application of EOF-ARIMA model in short-term prediction of VTEC in China was analyzed. The results showed that: (1) In Guangxi, the overall prediction effect of EOF-ARIMA model was better than that of ARIMA model: The root-mean-square error of EOF-ARIMA model in predicting 5-day VTEC values is $7.451 \mathrm{TECu}$, with an average relative accuracy of $84.0 \%$; the standard deviation of ARIMA model is $8.29 \mathrm{TECu}$, with an average relative accuracy of $84.5 \%$.(2) The prediction performance of EOF-ARIMA model has no obvious seasonal change, and the prediction accuracy of ARIMA model in autumn is lower than that of other seasons, indicating that the introduction of EOF decomposition into ARIMA model can improve the stability and accuracy of its VTEC short-term prediction.(3) Through the analysis of VTEC measured values of low, middle and high latitude grid points in China, it is found that VTEC values in low latitude areas of China have equatorial anomalies. Nevertheless, the average relative prediction accuracy of EOF-ARIMA model in different latitudes is still higher than $80 \%$, and the relative accuracy and standard deviation of VTEC prediction in the region of $5^{\circ} \sim 22.5^{\circ} \mathrm{N}$ do not show abnormal fluctuations, indicating that the short-term prediction performance of EOF-ARIMA model is not affected by ionospheric equatorial anomalies. The EOF-ARIMA model can maintain good accuracy and stability in short-term VTEC prediction of equatorial anomalies in Guangxi and China's low-latitude regions, and can be used in other applications such as GNSS navigation and positioning in Guangxi and the sea area south of Guangxi.

Thanks: thanks the IGS Center for providing the VTEC grid data!

\section{ACKNOWLEDGEMENTS}

This work was sponsored by the National Natural Foundation of China $(41664002 ; 41704027)$; Guangxi Natural Science Foundation of China(2018GXNSFAA294045;

2017GXNSFDA198016; 2017GXNSFBA198139); the "Ba Gui Scholars" program of the provincial government of Guangxi; and the Guangxi Key Laboratory of Spatial Information and Geomatics (14-045-24-10;16-380-25-01)

\section{REFERENCES}

Zhang Xuemin, Shen Xuhui, Zhao Shufan, et al. The seismo-ionospheric monitoring technologies and their application research development[J]. Acta Seismologica Sinica, 2016, 38(2): 365-375.

Lu Shikun, Li Yihong, Chang Wenlong, et al. Interactions between the spatio-temporal variations of the geomagnetic field and ionosphere during solar quiet periods[OL]. Progress in Geophysics, http://kns.cnki.net/kcms/detail/11.2982.P.20180725.11 18.060.html.

Liu Libo, Wan Weixing, Chen Wenlong, et al. The relationship between ionosphere and solar activity [J]. Chinese Science Bulletin, 2016, 56(7): 477-487

Mao Wenjun and Chang Wenlong. Prediction of ionospheric VTEC using grey time series combination model [J]. Science of Surveying and Mapping, 2016, 41(1): 39-43

Chen Biyan, Dai Wujiao, Cai Changshegn, et al. An application of time series and neural network method in ionospheric TEC prediction [J]. Geotechnical Investigation \& Surveying, 2011, 39(4): 64-68. 
Xie Shaofeng, Chen Jun, Huang Liangke, et al. Ionospheric TEC Prediction Based on Holt-Winters Models [J]. Journal of Geodesy and Geodynamics, 2017, 37(1): 72-76.

Liu Lilong, Chen Jun, Huang Liangke, et al. A sophisticated Klobuchar Model based on the Holt exponential smoothing model[J]. Geomatics and Information Science of Wuhan University, 2018, 43(4): 599-604.

Chen Peng, Yao Yibin, Wu Han, et al. TEC prediction of ionosphere based on time series analysis[J]. Geomatics and Information Science of Wuhan University, 2011, 36(3): 267-270.

Zhang Xiaohong, Ren Xiaodong, Wu Fengbo, et al. Short-term TEC prediction of ionosphere based on ARIMA model[J]. Acta Geodaetica et Cartographica Sinica, 2014, 43(2): 118-270.

Bao Yadong, Liu Changjian, Cai Hongzhou, et al. Time series prediction model of ionospheric VTEC improved by wavelet decomposition[J]. Journal of Geodesy and Geodynamics, 2015, 35(5): 784-787.

Pang Yishu, Zhu Congwen, Liu Kai, et al. Analysis of stability of EOF modes in summer rainfall anomalies in China[J]. Chinese Journal of Atmospheric Sciences, 2014, 38(6): 1137-1146.

Huang Wei, Zhang Deheng, Bao Yuanyuan, et al. Objective forecast of medium-term average temperature anomalies based on EOF technique $[\mathrm{J}]$. Journal of the Meteorological Sciences, 2017, 37(4): 561-566. doi: 10.3969/2016jms.0060.

$\mathrm{Wu}$ Yumiao. Investigation on tunnel deformation monitoring methods based on the EOF and neural network[D]. [Master dissertation], Southwest Jiaotong University, 2014.

Feng Jiandi, Wang Zhengtao, Shi Shuangshuang, et al. Using IGS to analyze the variation of anomaly equatorial ionization $[\mathrm{J}]$. Science of Surveying and Mapping, 2016, 41(6): 44-47,52.

Wang Pian, Qian Chuanhai, Zhang Ling, et al. The characteristics and impact of typhoon activities over western North Pacific and the South China Sea[J]. Journal of Marine Meteorology, 2018, 38(2): 1-11.

Sun Jinlong, Zhang Hengde, Bao Yuanyuan, et al. Activity and triggering mechanism of seismic belt along the northern South China Sea continental margin[J]. Journal of Tropical Oceanography, 2012, 31(3): 40-47.

Xu Jie, Deng Baichang, Yang Guanglin, et al. Study of the perturbations of ionosphere over south China associated with Heyuan earthquakes[J]. South China Journal of Seismology, 2017, 37(1): 1-7.
Mao Tian, Wang Jingsong, Bao Yuanyuan, et al. Effects of typhoon Matsa on ionospheric TEC[J]. Chinese Science Bulletin, 2009, 54(24): 3858-3863.

Yang Feng, Xue Bin, Liu Jian, et al. Rain attenuation prediction at $\mathrm{W}$ Band based on non-stationary time-series ARIMA model [J]. Journal of Electronics \& Information Technology, 2015, 37(10): 2475-2482.

Tian Xiangyu, Liu Lilong, Chen Jun, et al. Ionospheric TEC prediction based on combination model of ARIMA and Holt-winters[J]. Journal of Geomatics Science and Technology, 2018, 35(1): 44-48.

Huang Linfeng, Jiang Yong, Wang Jinsong, et al. Using IGS-TEC data to analyze the variation of EIA crest over south China region[J]. Chinese Journal of Space Science, 2015, 35(2): 152-158. 